\title{
A Novel Solution Based on Differential Evolution for Short-Term Combined Economic Emission Hydrothermal Scheduling
}

\author{
Chengfu Sun ${ }^{1}$, Songfeng $\mathrm{Lu}^{2}$ \\ School of Computer Science and Technology, \\ Huazhong University of Science and Technology, Wuhan, China \\ Email: ${ }^{1}$ ajason509@smail.hust.edu.cn, ${ }^{2}$ lusongfeng@sina.com \\ Received April 17, 2009; revised May 13, 2009; accepted May 18, 2009
}

\begin{abstract}
This paper presents a novel approach based on differential evolution for short-term combined economic emission hydrothermal scheduling, which is formulated as a bi-objective problem: 1) minimizing fuel cost and 2) minimizing emission cost. A penalty factor approach is employed to convert the bi-objective problem into a single objective one. In the proposed approach, heuristic rules are proposed to handle water dynamic balance constraints and heuristic strategies based on priority list are employed to repair active power balance constraints violations. A feasibility-based selection technique is also devised to handle the reservoir storage volumes constraints. The feasibility and effectiveness of the proposed approach are demonstrated and the test results are compared with those of other methods reported in the literature. Numerical experiments show that the proposed method can obtain better-quality solutions with higher precision than any other optimization methods. Hence, the proposed method can well be extended for solving the large-scale hydrothermal scheduling.
\end{abstract}

Keywords: Hydrothermal Power Systems, Economic Load Scheduling, Combined Economic Emission Scheduling, Differential Evolution

\section{Introduction}

One of the major problems existing today on electric power systems is the optimum scheduling of hydrothermal plants. Short-term hydrothermal scheduling is a daily planning task in power systems and its main objective is to minimize the total operational cost subjected to a variety of constraints of hydraulic and power system network. As the source for hydropower is the natural water resources, the operational cost of hydroelectric plants is insignificant. Thus, the objective of minimizing the operational cost of a hydrothermal system essentially reduces to minimize the fuel cost of thermal plants over a scheduling horizon while satisfying various constraints. Due to increasing concern over atmospheric pollution, harmful emission produced by the thermal units must be minimized simultaneously. So a revised economic power dispatch program considering both the fuel cost and emission is required. But minimizing pollution may lead to an increase in generation cost and vice versa.

The importance of the generation scheduling problem of hydrothermal systems is well recognized. Therefore, many methods have been devised to solve this difficult optimization problem for several decades. Some of these methods are dynamic programming methodology [1], linear programming [2], and decomposition techniques [3]. Recently, aside from the above methods, optimal hydrothermal scheduling problems have been solved by meta-heuristic approaches such as genetic algorithm [4-6], cultural algorithm [7] and particle swarm optimization [8] etc. Various heuristic methods such as heuristic search technique [9], fuzzy satisfying evolutionary programming procedures [10] and fuzzy decision-making stochastic technique [11] have been applied to solve 
multi-objective short-term hydrothermal scheduling problems. Because these meta-heuristic optimization methods are able to provide higher quality solutions, they have received more interest. One of these meta-heuristic optimization methods is differential evolution (DE) [13].

A new optimization method known as DE, which is a stochastic search algorithm based on population cooperation and competition of individuals, has gradually become more popular and has been successfully applied to solve optimization problems particularly involving non-smooth objective function. DE combines the simple arithmetic operators with the classical evolution operators of crossover, mutation and selection to evolve from a randomly generated population to a final solution. The DE algorithm has been applied to various fields of power system optimization such as dynamic economic dispatch with valve-point effects [14], hydrothermal scheduling [15], economic dispatch with non-smooth and non-convex cost functions [16], optimal reactive power planning in large-scale distribution system [17], and economic dispatch problem [18].

This work presents a novel approach based on differential evolution to solve short-term combined economic emission scheduling of cascaded hydrothermal systems. Moreover, heuristic rules are proposed to handle the water dynamic balance constraints and heuristic strategies based on priority list are employed to handle active power balance constraints. At the same time, a feasibility-based selection technique is devised to handle the reservoir storage volumes constraints. The results obtained with the proposed approach were analyzed and compared with the results of the differential evolution [12] and interactive fuzzy satisfying method based on evolutionary programming [10] reported in the literature.

The remainder of the paper is organized as follows. The formulation of the short-term combined economic emission scheduling of hydrothermal power systems with cascaded reservoirs is introduced in Section 2, while Section 3 explains the classical DE. Section 4 describes the implementation of the proposed method for solving the short-term hydrothermal scheduling and outlines heuristic strategies to handle water dynamic balance constraints and active power balance constraints. Section 5 presents the optimization results for the short-term hydrothermal power systems scheduling. Lastly, section 6 draws the conclusions.

\section{Problem Formulation}

The hydrothermal scheduling problem combined economic emission scheduling is formulated as a bi-objective optimization problem. It is concerned with the attempt to minimize the fuel cost and as well as the emission of thermal units, while making full use of the availability of hydro-resources as much as possible. In the formulation of the hydrothermal scheduling problem, the following objectives and constraints must be taken into account and the equality and inequality constraints must simultaneously be satisfied.

\subsection{Notations}

In order to formulate the hydrothermal scheduling problem mathematically, the following notations is introduced first:

$f_{i t}^{v}\left(P_{\text {sit }}\right)$ fuel cost of thermal plant $i$ including valve point loading

$e_{i t}^{v}\left(P_{\text {sit }}\right)$ emission of thermal plant $i$ including valve point loading

$a_{s i}, b_{s i}, c_{s i} e_{s i}, f_{s i}$ coefficients of thermal generating plant $i$

$\alpha_{s i}, \beta_{s i}, \gamma_{s i}, \eta_{s i} \delta_{s i}$ emission coefficients of thermal plant $i$

$T$ total time intervals over scheduling horizon

$N_{s}, N_{h}$ number of thermal and hydro plants respectively

$P_{h j t}$ power generation of hydro generating plant $j$ at time interval $t$

$P_{\text {sit }}$ power generation of thermal generating unit $i$ at time interval $t$

$P_{D t}$ power demand at time interval $t$

$P_{L t}$ total transmission loss at time interval $\mathrm{t}$

$C_{1 j}, C_{2 j}, C_{3 j}, C_{4 j}, C_{5 j}, C_{6 j}$ power generation coefficients of hydro plant $j$

$V_{h j t}$ storage volume of reservoir $j$ at time interval $t$

$Q_{h j t}$ water discharge rate of the $j$ th reservoir at time $t$.

$P_{s i}^{\min } \quad P_{s i}^{\max }$ minimum and maximum power generation by thermal plant $i$

$P_{s i}^{\min } P_{s i}^{\max }$ minimum and maximum power generation by hydro plant $j$

$V_{h j}^{\min }, V_{h j}^{\max }$ minimum and maximum storage volumes of reservoir $j$

$I_{h j t} \quad$ inflow of hydro reservoir $j$ at time interval $t$

$S_{h j t}$ spillage discharge rate of hydro plant $j$ at time interval $t$

$\tau_{m j}$ water transport delay from reservoir $m$ to $j$

$R_{u j}$ number of upstream hydro generating plants directly above reservoir $j$

$G$ current iteration generation

$N_{p}$ number of the parameter vectors

\subsection{Objective Functions}




\subsubsection{Economic Scheduling}

In this paper, non-smooth fuel cost function of thermal generating unit with valve-point effects is considered.

$$
f_{i t}^{v}\left(P_{s i t}\right)=a_{s i}+b_{s i} * P_{s i t}+c_{s i} * P_{s i t}^{2}+\left|e_{s i} * \sin \left\{f_{s i} *\left(P_{s i}^{\min }-P_{s i t}\right)\right\}\right|
$$

For a given hydrothermal system, the problem may be described as minimization of total fuel cost associated to the on-line $N$ units for $T$ intervals in the given time horizon as defined by Equation (2) under a set of operating constraints as follows:

$$
F=\min \sum_{t=1}^{T} \sum_{i=1}^{N_{s}}\left[f_{i t}^{v}\left(P_{\text {sit }}\right)\right]
$$

\subsubsection{Emission Scheduling}

In this study, the amount of emission from each generator can be described as the sum of a quadratic and an exponential function.

$$
e_{i t}^{v}\left(P_{s i t}\right)=\alpha_{s i}+\beta_{s i} * P_{s i t}+\gamma_{s i} * P_{s i t}^{2}+\eta_{s i} * \exp \left(\delta_{s i} * P_{s i t}\right)
$$

The economic emission scheduling problem can be expressed as the minimization of total amount of emission release defined by Equation(4) as

$$
E=\sum_{t=1}^{T} \sum_{i=1}^{N_{s}}\left[e_{i t}^{v}\left(P_{\text {sit }}\right)\right]
$$

\subsection{Constraints}

While minimizing the above two objectives, the following constraints must be satisfied simultaneously.

Active power balance constraint

$$
\sum_{i=1}^{N_{s}} P_{s i t}+\sum_{j=1}^{N_{h}} P_{h j t}-P_{D t}-P_{L t}=0
$$

The hydroelectric generation is a function of water discharge rate and reservoir water head, which can be expressed as follows:

$$
P_{h j t}=C_{1 j} * V_{h j t}^{2}+C_{2 j} * Q_{h j t}^{2}+C_{3 j} * V_{h j t} * Q_{h j t}+C_{4 j} * V_{h j t}+C_{5 j} * Q_{h j t}+C_{6 j}
$$

Generation limits constraints

$$
\begin{aligned}
& P_{s i}^{\min } \leq P_{s i t} \leq P_{s i}^{\max } \\
& P_{h j}^{\min } \leq P_{h j t} \leq P_{h j}^{\max }
\end{aligned}
$$

1) Reservoir storage volumes constraints

$$
V_{h j}^{\min } \leq V_{h j t} \leq V_{h j}^{\max }
$$

2) Discharge rates limit

$$
Q_{h j}^{\min } \leq Q_{h j t} \leq Q_{h j}^{\max }
$$

3) Water dynamic balance constraints

$$
V_{h j t}=V_{h j, t-1}+I_{h j t}-Q_{h j t}-S_{h j t}+\sum_{m=1}^{R_{u j}}\left(Q_{h m, t-\tau_{m j}}+S_{h m, t-\tau_{m j}}\right)
$$

\section{Overview of Differential Evolution Algorithm}

As a population-based and stochastic global optimizer, differential evolution (DE) is one of the latest evolutionary optimization methods proposed by Storn and Price [13]. In a DE algorithm, candidate solutions are randomly generated and evolved to final individual solution by simple technique combining simple arithmetic operators with the classical events of mutation, crossover and selection. One of the most frequently used mutation strategies, named "DE/rand/1/bin", will be employed in this paper.

\subsection{Mutation Operation}

The essential ingredient in the mutation operation is the vector difference. For each target vector $X_{i}^{G}\left(i=1,2, \cdots, N_{p}\right)$, the weighted difference between two randomly selected vectors $X_{l}^{G}$ and $X_{m}^{G}$ is added to a third randomly selected vector $X_{k}^{G}$ to generate a mutated vector $V_{i}^{G}$ using the following equation.

$$
V_{i}^{G}=X_{k}^{G}+F *\left(X_{l}^{G}-X_{m}^{G}\right)
$$

where $X_{k}^{G}, X_{l}^{G}$ and $X_{m}^{G}$ are randomly selected vectors and $i \neq k \neq l \neq m$; The mutation factor $F>0$ is a user chosen parameter to control the amplification of the difference between two individuals so as to avoid search stagnation.

\subsection{Crossover Operation}

Following the mutation phase, the crossover operation is performed in order to increase the diversity in the searching process.

$$
U_{i, j}^{G}= \begin{cases}V_{i, j}^{G} & \text { if }\left(\eta_{j} \leq C R\right) \text { or }(j=q) \\ X_{i, j}^{G} & \text { otherwise }\end{cases}
$$

where $\eta_{j} \in[0,1]$, generated anew for each value of $j$, is a uniformly distributed random number. The crossover factor $C R \in[0,1]$ controls the diversity of the population. $X_{i, j}^{G}, V_{i, j}^{G}$ and $U_{i, j}^{G}$ are the $j$ th parameter of the $i$ th target vector, mutant vector and trial vector at generation $G$, respectively.

\subsection{Selection Operation}


Thereafter, a selection operator is applied to compare the fitness function value of two competing vectors, namely, target and trial vectors to determine who can survive for the next generation.

$$
X_{i}^{G+1}= \begin{cases}U_{i}^{G} & \text { if } f\left(U_{i}^{G}\right) \leq f\left(X_{i}^{G}\right) \\ X_{i}^{G} & \text { otherwise }\end{cases}
$$

where $f$ denotes the fitness function under optimization (minimization).

\section{Implementation of the Proposed Method for Solving the Short-Term Hydrothermal Scheduling}

In this section, the procedures for solving short-term scheduling problem of hydrothermal power system are described in details. Especially, heuristic strategies will be given to handle constraints of hydrothermal scheduling problem. The process of the proposed method for solving hydrothermal scheduling can be summarized as follows.

\subsection{Structure of Parameter Solution Vector}

The structure of a solution for hydrothermal scheduling problem is composed of a set of decision variables which represent the discharge rate of the each hydro plant and the power generated by each thermal unit over the scheduling horizon.

$$
P_{k}=\left[\begin{array}{cccccccc}
Q_{h 11} & Q_{h 21} & \cdots & Q_{h N_{h} 1} & P_{s 11} & P_{s 21} & \cdots & P_{s N_{s} 1} \\
Q_{h 12} & Q_{h 22} & \cdots & Q_{h N_{h} 2} & P_{s 12} & P_{s 22} & \cdots & P_{s N_{s} 2} \\
\vdots & \vdots & \cdots & \vdots & \vdots & \vdots & \cdots & \vdots \\
Q_{h 1 T} & Q_{h 2 T} & \cdots & Q_{h N_{h} T} & P_{s 1 T} & P_{s 2 T} & \cdots & P_{s N_{s} T}
\end{array}\right]
$$

The elements $Q_{h j t}$ and $P_{\text {sit }} \quad P_{\text {sit }}\left(j=1,2 \cdots, N_{h} ; i=\right.$ $1,2, \cdots, N_{s}$ ) are subjected to the water discharge rate and the thermal generating capacity constraints as depicted in Equation. (10) and (7), respectively. The water discharge rate of the $j$ th hydro plant in the dependent interval must satisfy the water dynamic balance constraints in Equation (11).

\subsection{Initialization Parameter Vectors}

During the initialization process, the candidate solution of each parameter vector $X_{k}\left(k=1,2, \cdots, N_{p}\right)$ is randomly initialized within the feasible range as follows:

$$
\begin{gathered}
Q_{h j t}=Q_{h j}^{\min }+r_{q} *\left(Q_{h j}^{\max }-Q_{h j}^{\min }\right) \\
P_{s i t}=P_{s i}^{\min }+r_{s} *\left(P_{s i}^{\max }-P_{s i}^{\min }\right)
\end{gathered}
$$

where $r_{q}$ and $r_{s}$ are the random numbers uniformly distributed in $[0,1]$.

\subsection{Combined Economic and Emission Scheduling}

The short-term combined economic emission scheduling of hydrothermal power systems with cascaded reservoirs is a bi-objective problem with the attempt to minimize simultaneously fuel cost and emission of thermal plants. The bi-objective optimization problem can be transformed into a single objective one by introducing price penalty factors $h_{t}$. For more details, see Ref. [12].

\subsection{Solution Modification}

New values of water discharge rate $Q_{h j, t+1}$ and power generation $P_{s i, t+1}$ are generated through mutation and crossover operation according to Equations (12) and (13), respectively. The new values are not always guaranteed to satisfy the constraints Equations (10) and (7), respectively. If any value violating its constraint is modified in the following way:

$$
\begin{aligned}
Q_{h j, t+1} & =\left\{\begin{array}{lll}
Q_{h j}^{\min } & \text { if } \quad Q_{h j, t+1}<Q_{h j}^{\min } \\
Q_{h j, t+1} & \text { if } & Q_{h j}^{\min } \leq Q_{h j, t+1} \leq Q_{h j}^{\max } \\
Q_{h j}^{\max } & \text { if } & Q_{h j, t+1}>Q_{h j}^{\max }
\end{array}\right. \\
P_{s i, t+1} & =\left\{\begin{array}{lll}
P_{s i}^{\min } & \text { if } & P_{s i, t+1}<P_{s i}^{\min } \\
P_{s i, t+1} & \text { if } & P_{s i}^{\min } \leq P_{s i, t+1} \leq P_{s i}^{\max } \\
P_{s i}^{\max } & \text { if } & P_{s i, t+1}>P_{s i}^{\max }
\end{array}\right.
\end{aligned}
$$

\subsection{Heuristic Strategies to Handle Equality Constraints}

\subsubsection{Handling Water Dynamic Balance Constraints}

To meet exactly the restrictions on the initial and final reservoir storage, the water discharge rate of the $j$ th hydro plant in the dependent interval $d$ is then calculated using Equation(21). The dependent water discharge rate must satisfy the constraints in Equation (10). Assuming the spillage in Equation (11) to be zero for simplicity, the water dynamic balance constraints are

$$
V_{h j 0}-V_{h j T}=\sum_{t=1}^{T} Q_{h j t}-\sum_{t=1}^{T} \sum_{m=1}^{R_{u j}}\left(Q_{h m, t-\tau_{m j}}\right)-\sum_{t=1}^{T} I_{h j t}
$$


where $V_{h j 0}$ is the initial storage volume of reservoir $j$; $V_{h j T}$ is the final storage volume of reservoir $j$. The procedures for repairing the water dynamic balance violations in hydrothermal scheduling are as follows:

Step 1: Set $j=1$.

Step 2: Randomly choose a time interval $d$ as a dependent interval and set count $=1$.

Step 3: In order to meet equality constraint in Equation (11), the water discharge rate of the $j$ th hydro plant $Q_{h j d}$ in the dependent interval $d$ is then calculated by

$Q_{h j d}=V_{h j o}-V_{h j T}-\sum_{\substack{t=1 \\ t \neq d}}^{T} Q_{h j t}+\sum_{t=1}^{T} \sum_{m=1}^{R_{i j}}\left(Q_{h m, t-\tau_{m j}}\right)+\sum_{t=1}^{T} I_{h j t}$

If the computed $Q_{h j d}$ doesn't violate the constraints in Equation (10) then go to step 7; otherwise go to the next step

Step 4: Change $Q_{h j d}$ using Equation(18).

Step 5: A new random time interval $d$ is chosen ensuring that it is not repeatedly selected and count $=$ count +1 .

Step 6: If count $\leq T$, then go to step 3; otherwise go to next step.

Step 7: $j=j+1$, if $j \leq N_{h}$, then go to step 2; otherwise go to next step.

Step 8: The modification process is terminated.

\subsubsection{Handling Active Power Balance Constraints}

The power balance equality constraints in Equation(5) still remain to be resolved after the water dynamic balance constraints are preserved. The heuristic strategy based on priority list is proposed for handling the power balance constraints. In this paper, priority list is created according to each thermal plant parameter. When the thermal plant is at its maximum output power, the average full-load cost $\alpha_{i t}$ of thermal plant $i$ at time interval $t$ is defined by

$$
\alpha_{i t}=\frac{\omega_{1} * f_{i t}^{v}\left(P_{s i}^{\max }\right)+\omega_{2} * h_{t} * e_{i t}^{v}\left(P_{s i}^{\max }\right)}{P_{s i}^{\max }}
$$

where $h_{t}$ is price penalty factor at time interval $t$, $\omega_{1}$ and $\omega_{2}$ are the weight factors. The detail procedures for handling active power balance constraints are as follows:

Step 1: Calculate the average full-load cost $\alpha_{i t}$ using Equation(22) at time interval $t$. Arrange them in ascending order of $\alpha_{i t}$ to obtain a priority list $P L(t)$.

Step 2: Set $t=1$.

Step 3: Set temp_PL( $t)=P L(t)$.
Step 4: The amount of active power balance violation at time interval $t$ is calculated by $t=1 \Delta P^{t}=\sum_{i=1}^{N_{s}} P_{s i t}-\left(\sum_{j=1}^{N_{h}} P_{h j t}+P_{D t}\right)$. In this paper the power loss is not considered for simplicity.

Step 5: If $\Delta P^{t}=0$, go to Step 14; if $\Delta P^{t}>0$, go to Step 6; if $\Delta P^{t}<0$, go to Step 10 .

Step 6: Set $m=1$.

Step 7: Set power of the generator unit $k$ with highest $\alpha_{i t}$ in temp_PL(t) to be $P_{k}^{t}=P_{s k}^{\min }$.Then delete thermal unit $k$ from temp_PL(t).

Step 8: Calculate the total power $P_{\text {sum }}^{t}$ generated by all thermal units at time interval $t$. If $P_{\text {sum }}^{t} \leq \sum_{j=1}^{N_{h}} P_{h j t}+P_{D t}$, set $P_{k}^{t}=P_{s i}^{\min }+\left(\sum_{j=1}^{N_{h}} P_{h j t}+P_{D t}-P_{\text {sum }}^{t}\right)$ and go to step 14 ; otherwise set $P_{k}^{t}=P_{s k}^{\min }$.

Step 9: $m=m+1$. If $m \leq N_{s}$, then go to Step 7; otherwise go to Step 14

Step 10: Set $m=1$.

Step 11: Set power of the generator unit $k$ with lowest $\alpha_{i t}$ in temp $p_{-} P L(t)$ to be $P_{k}^{t}=P_{s k}^{\max }$. Then delete thermal unit $k$ from temp_PL(t).

Step 12: Calculate the total power $P_{\text {sum }}^{t}$ generated by all thermal units at time interval $t$. If $P_{\text {sum }}^{t} \geq \sum_{j=1}^{N_{h}} P_{h j t}+P_{D t}$, set $P_{k}^{t}=P_{s i}^{\max }+\left(\sum_{j=1}^{N_{h}} P_{h j t}+P_{D t}-P_{\text {sum }}^{t}\right)$ and go to step 14 ; otherwise set $P_{k}^{t}=P_{s k}^{\max }$.

Step 13: $m=m+1$.If $m \leq N_{s}$, then go to Step 11; otherwise go to Step 14 .

Step 14: $t=t+1$.If $t \leq T$, then go to Step 3; otherwise go to Step 15.

Step 15: The modification process is terminated.

\subsection{Selection Based Technique for Handling Reservoir Storage Volumes Constraints}

In this work, the feasibility-based selection rules are applied to the proposed approach for handling the inequality constraints of reservoir storage volumes constraints. The procedures for repairing the reservoir storage volumes constraints are as follows:

Step 1: The overall reservoir storage volumes constraints violation of solution $x$ is $C V(x)$, which is defined as 


$$
C V(x)=\sum_{t=1}^{T} \sum_{j=1}^{N_{h}}\left[\max \left(0, V_{h j t}-V_{h j}^{\max }, V_{h j}^{\min }-V_{h j t}\right)\right]
$$

Step 2: (1) If both parameter vectors are feasible, then the one with the better fitness value wins. (2) Otherwise, if both parameter vectors are infeasible, then the one with the less value of $C V(x)$ wins. (3) Otherwise, the feasible parameter vectors always wins.

\section{Simulation Results}

In this section, a test system consisting of a multi-chain cascade of four hydro units and three thermal units is studied to demonstrate the feasibility and effectiveness of the proposed method for solving short-term hydrothermal scheduling with cascaded reservoirs. The entire scheduling period is chosen as one day with 24 intervals of 1 hour each. The load demand of the system, hydro and thermal unit coefficients, reservoir inflows and reservoir limits are taken from the literature [10].

In order to compare with Ref. [12], the parameters for population size and maximum number of generations allowed are set as follows: $N_{p}=70$, maximum number of iterations Maxiter $=400$, respectively. Before proceeding to the simulated calculation, careful selection of mutation and crossover factor is important to produce a competent result. The following values for mutation and crossover factor were selected by parameter setting through trial and error for the present test system: mutation factor $F=0.44$, crossover factor $C R=0.85$. Under the chosen parameters, it has been found to provide optimum results. The proposed approach is performed 10 trials for different cases of hydrothermal scheduling.

According to [12], the total cost can be presented as follows for a trade off between fuel cost and emission cost.

$$
T C=\omega_{1} * F\left(P_{\text {sit }}\right)+\omega_{2} * h_{t} * E\left(P_{\text {sit }}\right)
$$

where $\omega_{1}$ and $\omega_{2}$ are the weight factors.

The results of proposed method for obtaining combined economic emission scheduling (CEES, $\omega_{1}=1$ and $\omega_{2}=1$ ) solution are illustrated as follows. In this case, the values $\alpha_{i t}$ of thermal unit 1,2 and 3 at time intervals $1,2,3,4,5,6,24$ are 4.8695, 6.2728 and 13.2897, while at other time intervals they are 7.1634, 11.8597 and 31.3219 . But the priority list is $\{1,2,3\}$ over the entire scheduling horizon. The thermal unit 1 with the lowest $\alpha_{i t}$ will have the highest priority to be dispatched more generation power. The optimal hydrothermal generation schedule for CEES is shown in Figure 1 and the optimal hourly water discharge rate obtained by the proposed method for CEES is presented in Figure 2.

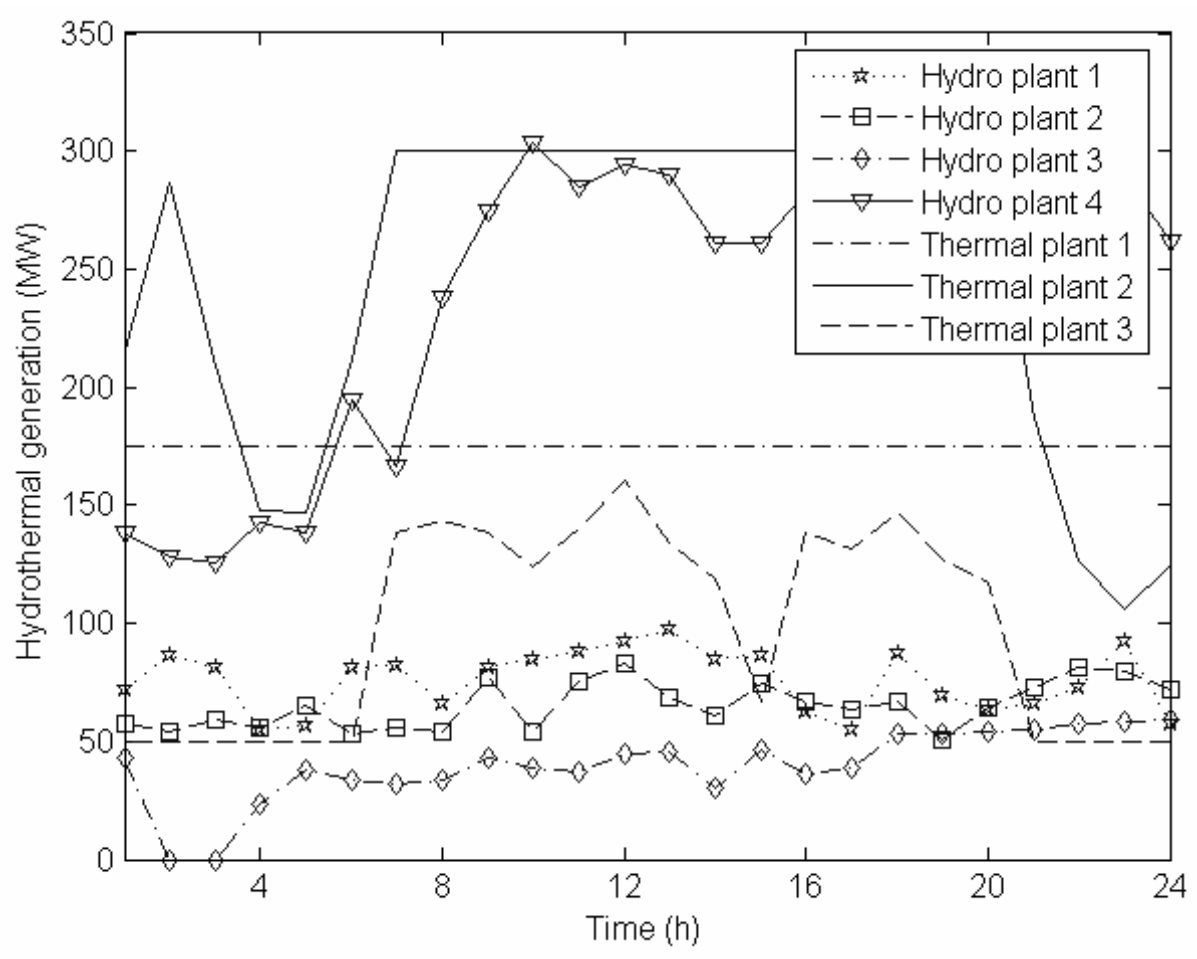

Figure 1. Hydrothermal generation (MW) schedule for CEES. 


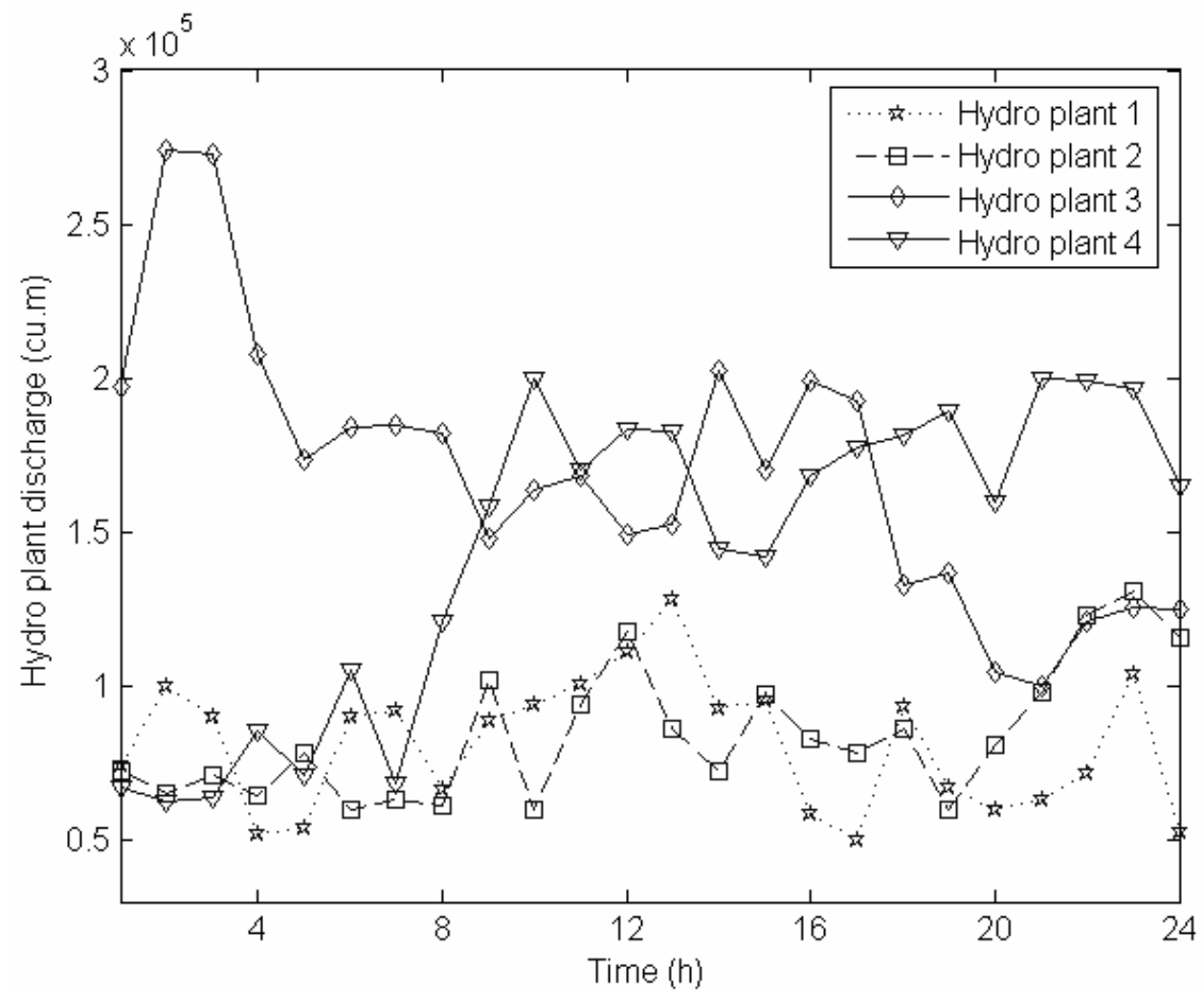

Figure 2. Hourly hydro plant discharge $\left(\mathrm{m}^{3}\right)$ for CEES.

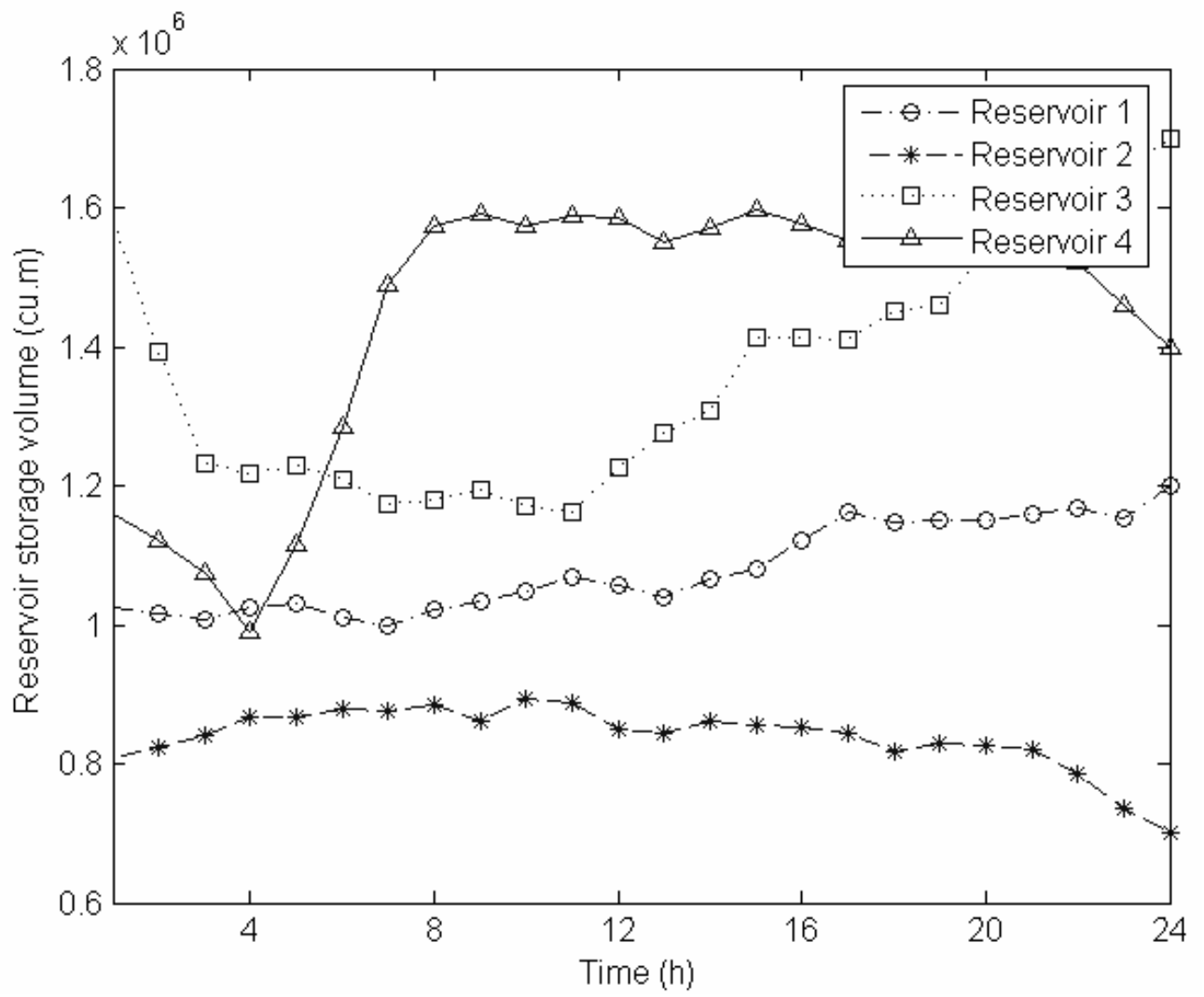

Figure 3. Reservoir storage volumes for CEES. 
The trajectories of reservoir storage volumes for CEES are shown in Figure 3. Table 1 shows that using the proposed method optimal fuel cost is found to be $\$ 44265.00$, while amount emission is found to be $18060.00 \mathrm{lb}$.

In Table 1, the optimal solutions of the fuel cost and emission cost for economic load scheduling (ELS, $\omega_{1}=1$ and $\omega_{2}=0$ ), economic emission scheduling (EES, $\omega_{1}=0$ and $\omega_{2}=1 / h_{t}$ ) and CEES obtained from the proposed approach have been compared with those of DE [12]. From the results it is quite evident that the proposed method provides better solutions for short-term combined economic emission hydrothermal scheduling with cascaded hydro reservoirs. Table 2 presents the best, worst and mean value of fuel cost and emission of CEES obtained by differential evolution without priority list, particle swarm optimization without priority list and the proposed approach. From the analysis of results in Table2, it can be seen that the proposed approach can produce valuable trade off solutions for CEES. It also shows that the two objectives of minimizing the fuel cost and emission cost are of conflicting nature, that is to say, minimizing pollution increases fuel cost and vice versa. From the results of CEES, it clearly sees that with some compromise in fuel cost, it is possible to obtain huge reduction in emission.

It can be seen clearly from Table 1 that the proposed method yields much better results in terms of fuel cost, the amount of emission than known optimization methods reported in the literature. It is also very important to note that compared with the results of fuzzy satisfying [10] the better results from [12] are obtained based on violating the constraints of the test system, such as the results of Table 1, Table 3 and Table 5 in Ref. [12], from which it is clearly shown that the power generation of thermal unit $P_{s 1}$ violates its constraint which is $20 \leq P_{s 1} \leq 175$ at some time intervals. However, in this study we obtain even better results while strictly satisfying all constraints of the test system.

\section{Conclusions}

In this paper, a novel approach in combination with novel equality constraint handling techniques has been successfully introduced to solve hydrothermal scheduling with non-smooth fuel and emission cost functions. The major advantages of this novel method are as follows: 1) In order to handle constraints effectively, heuristic rules are proposed to handle water dynamic balance constraints and heuristic strategies based on priority list are employed to handle active power balance constraints; 2)
The feasibility-based selection rules are developed to handle the reservoir storage volumes constraints. Additionally, the improved heuristic strategies can be simply incorporated into differential evolution. Hence the proposed method does not require the use of penalty functions and explores the optimum solution at a relatively lesser computational effort. Numerical experiments show that the proposed method can obtain better-quality solutions with higher precision than any other optimization methods reported in the literature. Hence, the proposed method can well be extended for solving the large-scale hydrothermal scheduling.

\section{Acknowledgements}

The authors gratefully acknowledge the financial supports from National Natural Science Foundation of China under Grant no. 10876012. The authors thank the anonymous Reviewers and Editors for constructive and detailed comments.

Table 1. Comparison of cost for ELS, EES and CEES by proposed method and DE.

\begin{tabular}{lccc}
\hline & & $\begin{array}{c}\text { Fuel cost } \\
(\$)\end{array}$ & $\begin{array}{c}\text { Emission } \\
(\mathrm{lb})\end{array}$ \\
\hline & ELS & 42766.00 & 31002.00 \\
The proposed method & EES & 46066.00 & 17655.00 \\
& CEES & 44265.00 & 18060.00 \\
& ELS & 43500.00 & 21092.00 \\
& & & \\
Differential evolution (DE) & EES & 51449.00 & 18257.00 \\
& & & \\
& CEES & 44914.00 & 19615.00 \\
\hline
\end{tabular}

Table 2. Comparison of cost of CEES by proposed method, DE and PSO.

\begin{tabular}{|c|c|c|c|c|}
\hline & & $\begin{array}{c}\text { Best } \\
\text { Value }\end{array}$ & $\begin{array}{l}\text { Worst } \\
\text { Value }\end{array}$ & $\begin{array}{l}\text { Mean } \\
\text { Value }\end{array}$ \\
\hline \multirow{2}{*}{$\begin{array}{c}\text { The proposed } \\
\text { method }\end{array}$} & Fuel cost(\$) & 44265 & 45258 & 44622 \\
\hline & Emission(lb) & 17797 & 18255 & 18069 \\
\hline \multirow{2}{*}{$\begin{array}{l}\text { Differential } \\
\text { evolution without } \\
\text { priority list }\end{array}$} & Fuel cost(\$) & 47999 & 48792 & 48390 \\
\hline & Emission(lb) & 17076 & 17716 & 17362 \\
\hline \multirow{2}{*}{$\begin{array}{l}\text { PSO without } \\
\text { priority list }\end{array}$} & Fuel cost $(\$)$ & 45670 & 46895 & 46530 \\
\hline & Emission(lb) & 16980 & 17807 & 17295 \\
\hline
\end{tabular}




\section{References}

[1] J. Tang and B. Peter, "Hydrothermal scheduling via extended differential dynamic programming and mixed coordination,” IEEE Transactions on Power System, Vol. 10, pp. 2021-2028, 1995.

[2] M. Piekutowski, "Optimal short-term scheduling for a large-scale cascaded hydro system,” IEEE Transactions on Power System, Vol. 9, pp. 805-811, 1994.

[3] M. V. F. Pereira and L. M. V. G. Pinto, “A decomposition approach to the economic dispatch of the hydrothermal systems,” IEEE Transactions on Power Systems, Vol. 101, pp. 3851-3860, 1982.

[4] M. Ramirez and P. E. Ontae, "The short-term hydrothermal coordination via genetic algorithms," Electric Power Components and Systems, Vol. 34, pp. 1-19, 2006.

[5] E. Gil, J. Bustos, and H. Rudnick, "Short-term hydrothermal generation scheduling model using a genetic algorithm,” IEEE Transactions on Power System, Vol. 18, pp. 1256-1264, 2003.

[6] X. Yuan and Y. Yuan, "A hybrid chaotic genetic algorithm for short-term hydro system scheduling," Mathematics and Computers in Simulation, Vol. 59, pp. 319-327, 2002.

[7] X. Yuan and Y. Yuan, "Application of cultural algorithm to generation scheduling of hydrothermal systems," Energy Conversion and Management, Vol. 47, pp. 21922201, 2006.

[8] B. Yu, X. Yuan, and J. Wang, "Short-term hydro-thermal scheduling using particle swarm optimization method," Energy Conversion and Management, Vol. 48, pp. 19021908, 2007.

[9] J. S. Dhillon and D.P. Kothari, "Multi-objective shortterm hydrothermal scheduling based on heuristic search technique,” Asian Journal of Information Technology, Vol. 6, pp. 447-454, 2007.

[10] M. Basu, “An interactive fuzzy satisfying method based on evolutionary programming technique for multi-objective short-term hydrothermal scheduling,” Electric Power Systems Research, Vol. 69, pp. 277-285, 2004.

[11] J. S. Dhillon, S. C. Parti, and D. P. Kothari, "Fuzzy decision-making in stochastic multi-objective short-term hydrothermal scheduling,” IEE Proceedings of Generation Transmission and Distribution, Vol. 149, pp. 191-200, 2002.

[12] K. K. Mandal and N. Chakraborty, "Short-term combined economic emission scheduling of hydrothermal power systems with cascaded reservoirs using differential evolution,” Energy Conversion and Management, Vol. 50, pp. 97-104, 2009.

[13] R. Storn and K. Price, "Differential evolution-a simple and efficient heuristic for global optimization over continuous spaces,” Journal of Global Optimization, Vol. 11, pp. 341-359, 1997.

[14] X. H. Yuan, L. Wang, Y. C. Zhang, and Y. B. Yuan, “A hybrid differential evolution method for dynamic economic dispatch with valve-point effects,” Expert System with Application, Vol. 36, pp. 4042-4048, 2009.

[15] X. H. Yuan, B. Cao, B. Yang, and Y. B. Yuan. "Hydrothermal scheduling using chaotic hybrid differential evolution”, Energy Conversion and Management, Vol. 49, pp. 3627-3633, 2008.

[16] S. K. Wang, J. P. Chiou, and C. W. Liu, "Non-smooth/ non-convex economic dispatch by a novel hybrid differential evolution algorithm,” IEE Proceeding Generation Transmission and Distribution, Vol. 1, pp. 793-803, 2007.

[17] C. F. Changa, J. J. Wong, J. P. Chiou, and C. T. Su, "Robust searching hybrid differential evolution method for optimal reactive power planning in large-scale distribution sytems," Electric Power Systems Research, Vol. 77, pp. 430-437, 2007.

[18] J. P. Chiou, "Variable scaling hybrid differential evolution for large-scale economic dispatch problems," Electric Power Systems Research, Vol. 77, pp. 212-218, 2007. 